\title{
Discovery of three nearby L dwarfs in the Southern Sky
}

\author{
N. Lodieu, R.-D. Scholz, and M. J. McCaughrean \\ Astrophysikalisches Institut Potsdam, An der Sternwarte 16, 14482 Potsdam, Germany
}

Received 22 April 2002 / Accepted 7 May 2002

\begin{abstract}
We report the discovery of three L dwarfs in the solar vicinity within 30 parsecs. These objects were originally found as proper motion objects from a combination of $R$ and $I$ photographic plates measured as part of the SuperCOSMOS Sky Surveys. We subsequently identified these objects as bona fide brown dwarf candidates on the basis of their $R-I$ colour, as first criterion, and subsequently their $J-K$ colours when the infrared data were available from the 2MASS database. Spectroscopic observations in the optical with the ESO $3.6 \mathrm{~m} / \mathrm{EFOSC} 2$ and in the near-infrared with the NTT/SOFI led to the classification of their spectral types as early L dwarfs.
\end{abstract}

Key words. surveys - stars: distances - stars: kinematics - stars: late-type - stars: low-mass, brown dwarfs solar neighbourhood

\section{Introduction}

The first L dwarf, Kelu-1, was discovered in the solar neighbourhood in 1997 (Ruiz et al. 1997). Up to now, more than $100 \mathrm{~L}$ dwarfs have been discovered mostly by DENIS, 2MASS, and SDSS all-sky surveys via optical spectroscopy based on their optical/infrared colours (Delfosse et al. 1997; Delfosse et al. 1999; Kirkpatrick et al. 1999; Reid et al. 2000; Fan et al. 2000; Schneider et al. 2002 and references therein). According to theoretical models (Burrows et al. 1997; Baraffe et al. 1998), most of them are expected to be brown dwarfs with effective temperature below $2000 \mathrm{~K}$ and ages of few Gyr. The substellar status of some late $\mathrm{M}$ and $\mathrm{L}$ dwarfs has been confirmed with subsequent lithium detections (Tinney et al. 1997; Delfosse et al. 1997; Tinney et al. 1998; Delfosse et al. 1999; Martín et al. 1999; Fan et al. 2000). All these objects are nearby, within $50 \mathrm{pc}$ as shown by parallax measurements and, therefore, exhibit significant proper motions. Three years ago, Scholz et al. (2000) started a new high proper motion survey in the Southern Sky using UK Schmidt plates spanning roughly $10-20$ years. The APM (Automatic Plate Measuring) photographic colours $\left(B_{J}\right.$ and $R$ ) have proven a useful discriminant of nearby lowmass objects straddling the stellar/substellar boundary (Kirkpatrick et al. 1997). Our selection criterion for very red and high proper motion objects provides a powerful tool for detecting low-luminosity objects, and, in this letter, we report the discovery of three early $\mathrm{L}$ dwarfs found

Send offprint requests to: N. Lodieu, e-mail: nlodieu@aip.de

* Based on observations collected with the ESO $3.6 \mathrm{~m} /$ EFOSC2 and the NTT/SOFI at the European Southern Observatory, La Silla, Chile (ESO programme 68.C-0664). via low-dispersion optical and near-infrared spectroscopy using ESO $3.6 \mathrm{~m} / \mathrm{EFOSC} 2$ and NTT/SOFI.

\section{Sample selection}

Brown dwarf candidates have been selected among extremely red objects discovered in different new high proper motion surveys in the southern sky. A detailed description of the different search methods and samples will be given in a forthcoming paper, but all are based on Schmidt plates scanned with various measuring machines. The three objects mentioned in this study were discovered from SuperCOSMOS Sky Surveys(SSS) data (Hambly et al. 2001a; Hambly et al. 2001b; Hambly et al. 2001c). $B_{J}$, $R$ and $I$ band measurements of UKST plates with additional $R$ band data from ESO Schmidt plates have been combined to search for proper motion objects.

The basic search strategy consisted of looking for objects on a given plate which were not matched with a corresponding object in a different passband to within a nominal search radius of 3 arcsec. This process was repeated for each available plate at a given location on the sky. These reduced catalogues of unmatched objects were then compared, looking for possible counterparts out to a search radius of 1 arcmin. Objects were identified as proper motion candidates if they were picked up at least three times along a straight line, to within errors on the implied proper motion of 30 mas/year. Later, further positional information from (e.g.) the 2MASS survey was used to refine these detections. The epoch differences between the plates vary from field to field, with typically a maximum of 15-20 years between the UKST $B_{J}$ and 
$R$ plates. The search was therefore sensitive to proper motions larger than $0.15-0.20 \mathrm{arcsec} / \mathrm{yr}$.

As all three objects were discovered in SSS data, they have correspondingly been named SSSPM J0219-1939, SSSPM J2310-1759, and SSSPM J0109-5101. The first two objects were also identified on the POSS-I plates also measured as part of the SSS (although not on the UKST $B_{J}$ plates), and in the 2MASS 2nd incremental release public data base. No 2MASS measurements are presently available for SSSPM J0109-5101, and thus its $K_{\mathrm{s}}$ magnitude was derived from the SOFI acquisition image. Table 1 lists the data obtained from SSS and 2MASS. The proper motions of the first two objects given in Table 1 are based on the positions on two $R$ and one $I$ band plates, as well as on the additional SSS measurements of the POSS-I plates and on the 2MASS positions. The proper motion of SSSPM J0219-1939 was obtained from $B_{J}, R$, and $I$ band measurements of UKST plates. Positions are given at the latest available epoch.

\section{Observations and data reduction}

\subsection{ESO3.6 m/EFOSC2}

Spectroscopy was first obtained in the optical for SSSPM J0219-1939 and SSSPM J2310-1759 with EFOSC2 mounted on the ESO $3.6 \mathrm{~m}$ telescope at La Silla on 22 November 2001. The night was photometric and the seeing around $0.6-0.8$ arcsec. The camera uses a $2048 \times 2048$ pixels Loral/Lesser CCD. The pixel size is 0.157 arcsec and the useful field of view is $5.2 \times$ 5.2 arcmin. A 1 arcsec slit has been used for spectroscopy with Grism 12 covering 6000-10300 A, yielding a resolution of $\sim 600$. Three exposures of $1200 \mathrm{~s}$ each, shifted along the slit by 30 arcsec have been obtained for both candidates. The data reduction consisted in subtracting an averaged dark frame and dividing by an internal quartz flat field taken just after the first exposure of the target in order to remove efficiently fringing above $8000 \AA$. Wavelength calibration was made using $\mathrm{He}$ and Ar lines throughout the whole wavelength range. Flux calibration was achieved using an averaged sensitivity function determined with several exposures of the spectrophotometric standard stars EG21 and LTT1788 during the night. The co-added optical spectra of SSSPM J0219-1939 and SSSPM J2310-1759 are shown in Fig. 1. The TiO and VO absorption bands and ground-state transitions of the alkali elements including $\mathrm{NaI}$ and KI doublets, are labelled on each spectrum, along with the locations of the lithium and $\mathrm{H} \alpha$ lines at $6708 \AA$ and $6563 \AA$, respectively. Furthermore, a higher resolution spectrum of the lithium and $\mathrm{H} \alpha$ region of SSSPM J0219-1939 has been obtained with the Grism 10 (6280-8200 $)$ on ESO 3.6 m/EFOSC2 in January 2002 yielding a dispersion of $1 \AA$. Data reduction was standard and similar to the one applied for Grism 12 described previously. A zoom of the lithium and $\mathrm{H} \alpha$ region of SSSPM J0219-1939 is plotted in the left upper corner of Fig. 1.

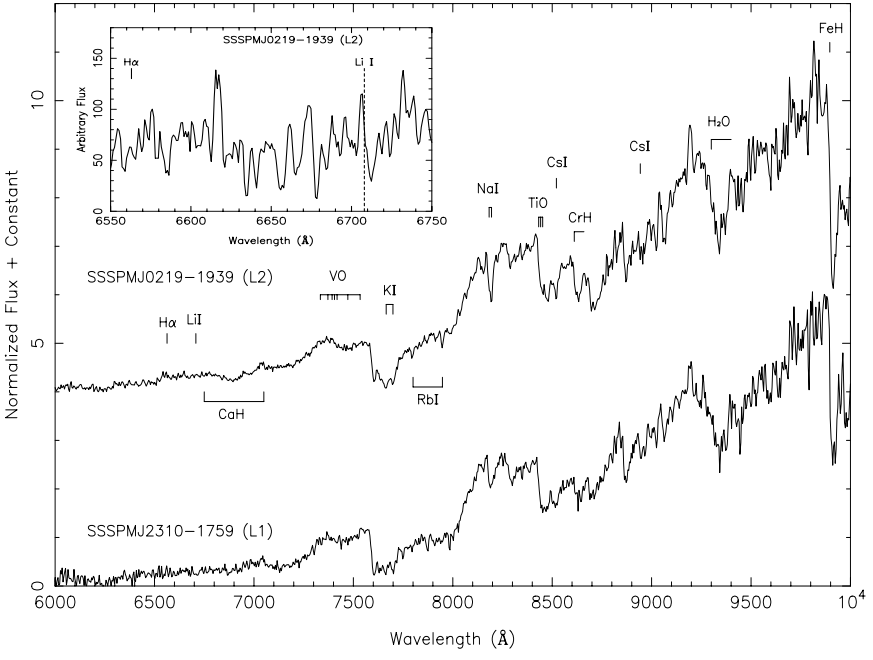

Fig. 1. Low-resolution $(R \sim 600)$ optical spectra of SSSPM J0219-1939 (top) and SSSPM J2310-1759 (bottom) using the Grism 12 on the $3.6 \mathrm{~m} / \mathrm{EFOSC} 2$. An arbitrary constant has been added to separate the spectra. Their spectral types are estimated to be L2 and L1, respectively (see text). Labelled are the $\mathrm{TiO}$ and $\mathrm{VO}$ absorption bands, alkali elements and hydride bands, as well as the locations of lithium at $6708 \AA$ and $\mathrm{H} \alpha$ at $6563 \AA$.

\subsection{NTT/SOFI}

Initial assessment of our optical spectra immediately identified these two sources as early L dwarfs. SSSPM J0219-1939 and SSSPM J2310-1759 have been followed up at longer wavelengths $(0.95-2.5 \mu \mathrm{m})$ with the near-infrared camera/spectrograph SOFI mounted on the New Technology Telescope at La Silla on 24 November 2001. SOFI is equipped with a $1024 \times 1024$ pixels $\mathrm{HgCdTe}$ (HAWAII) array (Moorwood \& Spyromilio 1999) with a pixel size of 0.294 arcsec for the Large Field Objective used for spectroscopy. The third object in our present sample, SSSPM J0109-5101, was selected on the basis of its $R-I$ colour alone and observed on 25 November 2001 with the same instrument configuration. Both NTT nights were photometric and the seeing variable from $0.8-$ 1.0 arcsec. A 1 arcsec slit was used yielding $R \sim 600$ for both blue $(0.95-1.64 \mu \mathrm{m})$ and red $(1.53-2.52 \mu \mathrm{m})$ gratings. Featureless spectrophotometric standards (typically F5-F7) were measured within 1 degree on the sky to remove telluric absorption and flux calibrate. Three positions along the slit have been made with $250 \mathrm{~s}$ each for SSSPM J0219-1939 and SSSPM J2310-1759 and $60 \mathrm{~s}$ each for SSSPM J0109-5101 to subtract the sky. Each individual frame has been flat-fielded, sky-subtracted and a one-dimensional spectrum extracted. This spectrum has then been divided by the spectrophotometric standard and multiplied by a template smoothed to our resolution, whose spectral type is identical to the observed standard. Infrared spectra for the three L dwarfs are shown in Fig. 2, along with the locations of $\mathrm{H}_{2} \mathrm{O}$ and $\mathrm{CO}$ bands and atomic lines. 
Table 1. Astrometry and photometry from SSS and 2MASS. 2MASS infrared magnitudes are not available for SSSPM J0109-5101. ${ }^{a} K_{\mathrm{s}}$ photometry was computed from the SOFI acquisition images.

\begin{tabular}{|c|c|c|c|c|c|c|c|c|c|}
\hline $\begin{array}{c}\text { Name } \\
\operatorname{SSSPM} J \ldots\end{array}$ & $\begin{array}{c}\alpha, \delta \\
(\mathrm{J} 2000)\end{array}$ & Epoch & $\begin{array}{r}\mu_{\alpha} \cos \delta \\
\operatorname{mas}\end{array}$ & yr & $\begin{array}{l}R \\
(\mathrm{~S}\end{array}$ & I) $I$ & $\overline{\bar{J}}$ & $\begin{array}{c}H \\
\text { (2MASS }\end{array}$ & 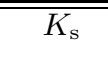 \\
\hline $0219-1939$ & $021928.03-193841.0$ & 1999.87 & $+194 \pm 04$ & $-173 \pm 06$ & 20.13 & 17.46 & $\overline{14.09}$ & 13.30 & 12.83 \\
\hline $2310-1759$ & $2310 \quad 18.53-175909.4$ & 1998.10 & $+24 \pm 17$ & $-246 \pm 13$ & 20.52 & 17.67 & 14.40 & 13.58 & 13.01 \\
\hline $0109-5101$ & 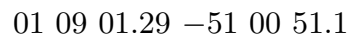 & 1990.80 & $+207 \pm 04$ & $+94 \pm 11$ & 18.21 & 14.80 & & & $10.96^{a}$ \\
\hline
\end{tabular}

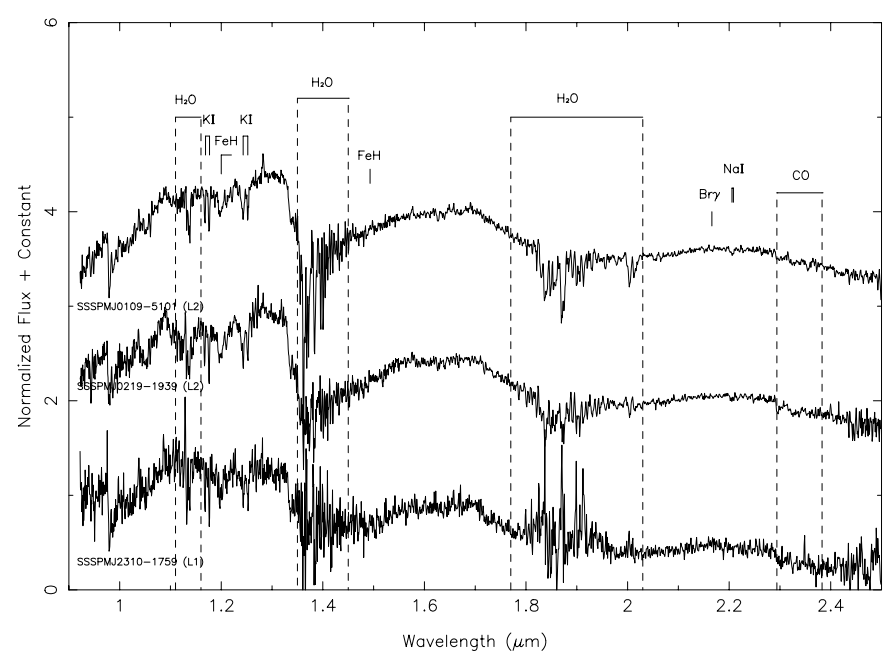

Fig. 2. Near-infrared spectra of SSSPM J2310-1759, SSSPM J0219-1939 and SSSPM J0109-5101 from bottom to top. An arbitrary constant has been added to separate the spectra. The locations of prominent bands of $\mathrm{H}_{2} \mathrm{O}, \mathrm{FeH}$ and $\mathrm{CO}$ are labelled, as well as atomic lines including $\mathrm{K} \mathrm{I}$ and $\mathrm{Na}$ I.

\section{Results and discussion}

\subsection{Spectral type classification}

The optical spectra of SSSPM J0219-1939 and SSSPM J2310-1759 reveal features typical of early $\mathrm{L}$ dwarfs. The $\mathrm{TiO}$ and $\mathrm{VO}$ absorption bands are weaker than those found in M dwarfs. KI and NaI doublets are also clearly seen although the latter is barely resolved at this low resolution. Hydride bands including $\mathrm{CrH}$, FeH and $\mathrm{CaH}$ as well as other alkali elements including Cs I and RbI clearly emerge from the optical spectra shown in Fig. 1. Furthermore, $\mathrm{H} \alpha$ is weakly seen in both objects which probably means that our targets were observed in a quiet state since a $\mathrm{H} \alpha$ flare has been recently reported in a L3 dwarf (Hall 2002). Moreover, a higher resolution spectrum of SSSPM J0219-1939 has been obtained in January 2002 using the Grism 10 (6280-8200 $)$ on ESO $3.6 \mathrm{~m} / \mathrm{EFOSC} 2$ to search for lithium at $6708 \AA$ (see Fig. 1). However, no significant absorption line is detected at this location which set the lower mass limit of this object at $0.065 M_{\odot}$. After the discovery of several objects cooler than M dwarfs, two spectral type schemes have been defined by Kirkpatrick et al. (1999) and Martín et al. (1999) to classify late $\mathrm{M}$ and L dwarfs. On the one hand, Kirkpatrick et al. (1999) considers the median spectral type value computed for several ratios of alkali elements and oxide absorption bands (see Table 2). On the other hand, the PC3 index is found to be the most reliable index among those defined by Martín et al. (1999) to classify $\mathrm{M}$ and $\mathrm{L}$ dwarfs. Computations of the different ratios mentioned above led to consistent results for SSSPM J0219-1939 and SSSPM J2310-1759 whose spectral types have been estimated to be L2 and L1, respectively.

Infrared spectra of the three objects quoted in this study denote strong $\mathrm{H}_{2} \mathrm{O}$ absorption bands, the $\mathrm{CO}$ break around $2.3 \mu \mathrm{m}$, and strong atomic lines including $\mathrm{KI}$, features characteristic of L dwarfs (see Fig. 2). Recently, several infrared indices have been defined mostly based on the strength of water absorption bands (Martín et al. 1999; Tokunaga \& Kobayashi 1999; Reid et al. 2001; Testi et al. 2001; Geballe et al. 2002; Martín 2000). The name of each index is given in Table 2 and the definition refers to the papers quoted above. Most of the spectral type computations are internally consistent yielding L1 except the one defined by Tokunaga \& Kobayashi (1999) and Reid et al. (2001) where discrepancies are noticed. As the optical classifications are, up to now, more accurate than the infrared schemes, spectral types of L1 and L2 are kept for SSSPM J2310-1759 and SSSPM J0219-1939, respectively. In the case of SSSPM J0109-5101, comparison of the infrared spectra plotted in Fig. 2 shows that this object is later than SSSPM J2310-1759 and similar to SSSPM J0219-1939. A spectral type of L2 is therefore adopted with an uncertainty of a subclass. Meanwhile, the analysis of the $K$ band spectra via two indices defined by Tokunaga \& Kobayashi (1999) provide a rough estimate of the effective temperature spanning 1800-2000 K for our objects. As the authors claim that brown dwarfs are likely to have $K 1 \geq 0.250$, the computed values for our objects are clearly below and therefore they are probably not brown dwarfs although SSSPM J0109-5101 has the highest probability to lie in the substellar regime. A high-resolution optical spectrum of this object is required to check this assessment.

\subsection{Distance estimates}

Assuming the spectral type determined above, distances can be computed using fiducial L dwarfs with known absolute magnitudes and trigonometric parallaxes (Kirkpatrick et al. 2000). Distances of $26.7 \mathrm{pc}$ and 30.8 pc have been computed for SSSPM J2310-1759 and SSSPM J0219-1939, respectively. Photometry of 
Table 2. Optical and near-infrared indices and corresponding spectral type for the three candidates (ST). Letters in brackets refer to the following references. Martín et al. (1999) [a], Kirkpatrick et al. (1999) [b], Tokunaga \& Kobayashi (1999) [c], Reid et al. (2001) [d], Testi et al. (2001) [e], Geballe et al. (2001) [f] and Martín (2000) [g].

\begin{tabular}{lccc}
\hline \hline Ref \& Feature & Value (ST) & Value (ST) & Value (ST) \\
SSSPM J.. & $0219-1939$ & $2310-1759$ & $0109-5101$ \\
\hline [a] PC3 & $3.05(\mathrm{~L} 2)$ & $2.30(\mathrm{~L} 1)$ & \\
[b] CrH-a & $1.36(\mathrm{~L} 1)$ & $1.17(\mathrm{~L} 0)$ & \\
[b] Rb-b/TiO-b & $1.10(\mathrm{~L} 2-\mathrm{L} 3)$ & $0.47(?)$ & \\
[b] Cs-a/VO-b & $1.13(\mathrm{~L} 3)$ & $0.99(\mathrm{~L} 2)$ & \\
\hline [c] $\mathrm{K} 1$ & $0.158(\mathrm{~L} 1)$ & $0.128(\mathrm{~L} 0)$ & $0.223(\mathrm{~L} 2)$ \\
[c] $\mathrm{K}$ & -0.01 & -0.060 & -0.010 \\
[d] $\mathrm{H}_{2} \mathrm{O}^{\mathrm{A}}$ & $0.60(\mathrm{~L} 4)$ & $0.72(\mathrm{~L} 0)$ & $0.66(\mathrm{~L} 2)$ \\
[d] $\mathrm{H}_{2} \mathrm{O}^{\mathrm{B}}$ & $0.69(\mathrm{~L} 3)$ & $0.78(\mathrm{~L} 1)$ & $0.77(\mathrm{~L} 1.5)$ \\
[e] $\mathrm{sHJ}^{\mathrm{e}] \mathrm{sKJ}}$ & $0.45(\mathrm{~L} 1)$ & $0.35(\mathrm{~L} 1)$ & $0.33(\mathrm{~L} 1)$ \\
[e] $\mathrm{sH}_{2} \mathrm{O}^{\mathrm{J}}$ & $0.91(\mathrm{~L} 1)$ & $0.94(\mathrm{~L} 1)$ & $0.79(\mathrm{~L} 1)$ \\
[e] $\mathrm{sH}_{2} \mathrm{O}^{\mathrm{H} 1}$ & $0.18(\mathrm{~L} 1)$ & $-0.07(\mathrm{~L} 1)$ & $0.21(\mathrm{~L} 1)$ \\
[e] $\mathrm{sH}_{2} \mathrm{O}^{\mathrm{H} 2}$ & $0.40(\mathrm{~L} 1)$ & $0.22(\mathrm{~L} 1)$ & $0.29(\mathrm{~L} 1)$ \\
[e] $\mathrm{sH}_{2} \mathrm{O}^{\mathrm{K}}$ & $0.16(\mathrm{~L} 1)$ & $0.39(\mathrm{~L} 1)$ & $0.36(\mathrm{~L} 1)$ \\
[f] $\mathrm{H}_{2} \mathrm{O} 1.5 \mu \mathrm{Lm}$ & $1.49(\mathrm{~L} 1)$ & $0.14(\mathrm{~L} 1)$ & $0.18(\mathrm{~L} 1)$ \\
[g] $\mathrm{QH}_{\mathrm{H}-\text { band }}$ & $0.41(\mathrm{~L} 1)$ & $0.37(\mathrm{~L} 2)$ & $01.30(\mathrm{~L} 1)$ \\
\hline
\end{tabular}

SSSPM J0109-5101 from the acquisition images yields $K_{\mathrm{s}}=10.96$, roughly one magnitude brighter than Kelu-1, provides a distance of 13 pc assuming a distance of 19.2 pc for Kelu-1 (Kirkpatrick et al. 2000). Then, the tangential velocities of these objects have been computed using classical formulae and velocities of $14.0 \mathrm{~km} \mathrm{~s}^{-1}, 32.6 \mathrm{~km} \mathrm{~s}^{-1}$ and $40.3 \mathrm{~km} \mathrm{~s}^{-1}$ derived for SSSPM J0109-5101, SSSPM J2310-1759, and SSSPM J0219-1939, respectively. These velocities are consistent with the kinematics of disk stars.

\section{Conclusions}

Three nearby L dwarfs have been discovered in a new high proper motion survey of the Southern Sky. These objects are members of a larger sample of red and nearby proper motion candidates which will be described in a forthcoming paper. Spectral types have been estimated to be L1, L2 and L2 for SSSPM J2310-1759, SSSPM J0219-1939, and SSSPM J0109-5101, respectively. Optical and infrared classification schemes led to consistent results within a subclass although some discrepancies are noticed depending on the index used. These three $\mathrm{L}$ dwarfs lie within $30 \mathrm{pc}$ and have velocities consistent with disk components. Further observations are required, first, to improve distance estimates and, second, to assess their masses and thus potential brown dwarf status via high-resolution spectroscopy enabling lithium detection.

Acknowledgements. The authors acknowledge enriching discussion with the support teams during the different runs. We also thank Martin Kürster, George Hau and Gaspare Lo-Curto for taking the higher resolution spectra of SSSPM J0219-1939. The discovery of the faint high proper motion objects is based on the SuperCOSMOS Sky Surveys, i.e. digitized data obtained from scans of UKST and ESO Schmidt plates. We would like to thank the SuperCOSMOS team for producing such excellent data. This research has also made use of data products from the Two Micron All Sky Survey, which is a joint project of the University of Massachusetts and the Infrared Processing and Analysis Center, funded by the National Aeronautics and Space Administration and the National Science Foundation. NL thanks the European Research Training Network on "The Formation and Evolution of Young Stellar Clusters" (HPRNCT-2000-00155) for financial support.

\section{References}

Baraffe, I., Chabrier, G., Allard, F., et al. 1998, A\&A, 337, 403 Burrows, A., Marley, M., Hubbard, W. B., et al. 1997, ApJ, 491,856

Delfosse, X., Tinney, C. G., Forveille, T., et al. 1997, A\&A, 327, L25

Delfosse, X., Tinney, C. G., Forveille, T., et al. 1999, A\&A, 135,41

Delfosse, X., Forveille, T., Martín, E. L., et al. 2001, A\&A, 366, L13

Fan, X., Knapp, G. R., Strauss, M. A., et al. 2000, AJ, 119, 928

Geballe, T. R., Knapp, G. R., Leggett, S. K., et al. 2002, ApJ, 564,466

Hall, P. B. 2002, ApJ, 564, 89

Hambly, N. C., MacGillivray, H. T., Read M. A., et al. 2001a, MNRAS, 326, 1279

Hambly, N. C., Irwin, M. J., \& MacGillivray, H. T. 2001b, MNRAS, 326, 1295

Hambly, N. C., Davenhall, A. C., Irwin, M. J., \& MacGillivray, H. T. 2001c, MNRAS, 326, 1315

Henry T. J., Ianna, P. A., Kirkpatrick, J. D., et al. 1997, AJ, 114,388

Kirkpatrick, J. D., Henry, T. J., \& Irwin, M. J. 1997, AJ, 113, 1421

Kirkpatrick, J. D., Reid, I. N., Liebert, J., et al. 1999, ApJ, 519,802

Kirkpatrick, J. D., Reid, I. N., Liebert, J., et al. 2000, AJ, 120, 447

Martín, E. L., Delfosse, X., Basri, G., et al. 1999, AJ, 118, 2466 Martín, E. L. 2000, in Very Low-mass Stars and Brown Dwarfs, ed. R. Rebolo, \& M. R. Zapatero Osorio (Cambridge University Press), 153

Moorwood, A. F. M., \& Spyromilio, J. 1997, in The Early Universe with the VLT, ed. J. Bergeron (Springer), 21

Reid, I. N., Kirkpatrick, J. D., Gizis, J. E., et al. 2000, AJ, 119,369

Reid, I. N., Burgasser, A. J., Cruz, K. L., et al. 2001, AJ, 121, 1710

Ruiz, M. T., Leggett, S. K., \& Allard, F. 1997, ApJ, 491, 107 Schneider, D. P., Knapp, G. R., Hawley, S. L., et al. 2002, AJ, 123,458

Scholz, R.-D., Irwin, M., Ibata, R., Jahreiß, H., \& Malkov, O. Yu. 2000, A\&A, 353, 958

Testi, L., D'Antona, F., Ghinassi, F., et al. 2001, ApJ, 552, 147

Tinney, C. G., Delfosse, T., \& Forveille, T. 1997, ApJ, 490, 95

Tinney, C. G., Delfosse, T., \& Forveille, T., et al. 1998, A\&A, 338,1066

Tokunaga, A., \& Koyabashi, N. 1999, AJ, 117, 1010 